\title{
Development of heart block in children of SSA/SSB-autoantibody-positive women is associated with maternal age and displays a season-of-birth pattern
}

\author{
Aurelie Ambrosi, Stina Salomonsson, Håkan Eliasson, Elisabeth Zeffer, Amanda Skog, \\ Vijole Dzikaite, Gunnar Bergman, Eva Fernlund, Joanna Tingström, Elke Theander, \\ Annika Rydberg, Thomas Skogh, Annika Öhman, Ulla Lundström, Mats Mellander, \\ Ola Winqvist, Michael Fored, Anders Ekbom, Lars Alfredsson, Henrik Källberg, \\ Tomas Olsson, Fredrik Gadler, Anders Jonzon, Ingrid Kockum, \\ Sven-Erik Sonesson and Marie Wahren-Herlenius
}

\section{Linköping University Post Print}

N.B.: When citing this work, cite the original article.

Original Publication:

Aurelie Ambrosi, Stina Salomonsson, Håkan Eliasson, Elisabeth Zeffer, Amanda Skog, Vijole Dzikaite, Gunnar Bergman, Eva Fernlund, Joanna Tingström, Elke Theander, Annika Rydberg, Thomas Skogh, Annika Öhman, Ulla Lundström, Mats Mellander, Ola Winqvist, Michael Fored, Anders Ekbom, Lars Alfredsson, Henrik Källberg, Tomas Olsson, Fredrik Gadler, Anders Jonzon, Ingrid Kockum, Sven-Erik Sonesson and Marie Wahren-Herlenius, Development of heart block in children of SSA/SSB-autoantibody-positive women is associated with maternal age and displays a season-of-birth pattern, 2012, Annals of the Rheumatic Diseases, (71), 3, 334-340.

http://dx.doi.org/10.1136/annrheumdis-2011-200207

Copyright: BMJ Publishing Group

http://group.bmj.com/

Postprint available at: Linköping University Electronic Press

http://urn.kb.se/resolve?urn=urn:nbn:se:liu:diva-75459 


\section{Development of heart block in SSA/SSB autoantibody-positive pregnancies is associated with maternal age and display a season-of-birth pattern}

Aurélie Ambrosi $\mathrm{PhD}^{* 1}$, Stina Salomonsson $\mathrm{PhD}^{* 1}$, Håkan Eliasson $\mathrm{MD}^{2}$, Elisabeth Zeffer $\mathrm{RN}^{1}$,

Vijole Dzikaite $\mathrm{PhD}^{1}$, Gunnar Bergman $\mathrm{MD}, \mathrm{PhD}^{2}$, Eva Fernlund $\mathrm{MD}^{3}$, Elke Theander MD, $\mathrm{PhD}^{4}$, Annika Rydberg MD, $\mathrm{PhD}^{5}$, Thomas Skogh MD, $\mathrm{PhD}^{6}$, Annika Öhman $\mathrm{MD}^{7}$, Ulla

Lundström $\mathrm{MD}^{8}$, Mats Mellander $\mathrm{MD}, \mathrm{PhD}^{8}$, Ola Winqvist $\mathrm{MD}, \mathrm{PhD}^{1}$, Michael Fored MD, $\mathrm{PhD}^{1}$, Anders Ekbom MD, $\mathrm{PhD}^{1}$, Lars Alfredsson $\mathrm{PhD}^{9}$, Henrik Källberg $\mathrm{PhD}^{9}$, Tomas Olsson $\mathrm{MD}, \mathrm{PhD}^{10}$, Fredrik Gadler $\mathrm{MD}, \mathrm{PhD}^{1}$, Anders Jonzon $\mathrm{MD}, \mathrm{PhD}^{7}$, Ingrid Kockum $\mathrm{PhD}^{10}$, SvenErik Sonesson MD, $\mathrm{PhD}^{2}$, Marie Wahren-Herlenius $\mathrm{MD}, \mathrm{PhD}^{1}$

${ }^{1}$ Department of Medicine, ${ }^{2}$ Department of Women and Child Health, Karolinska Institutet, Stockholm, ${ }^{3}$ Department of Pediatric Cardiology, Skåne University Hospital, Lund, ${ }^{4}$ Department of Rheumatology, Skåne University Hospital, Malmö, ${ }^{5}$ Department of Clinical Sciences, Paediatrics, Umeå University Hospital, Umeå, ${ }^{6}$ Rheumatology/AIR, Clinical and Experimental Medicine, Linköping University, Linköping, ${ }^{7}$ Department of Women's and Children's Health, Section for Pediatrics, Uppsala University, Uppsala, ${ }^{8}$ Department of Pediatric Cardiology, The Queen Silvia Children's Hospital, Sahlgrenska University Hospital, Göteborg, ${ }^{9}$ Institute of Environmental Medicine, ${ }^{10}$ Department of Clinical Neuroscience, Karolinska Institutet, Stockholm, Sweden

* These authors contributed equally.

\section{Corresponding author:}

Marie Wahren-Herlenius 
Rheumatology Unit, Department of Medicine

Karolinska Institutet, 17176 Stockholm, Sweden

Phone: +46-8-51773431, Fax: +46-8-51775562

Email: marie.wahren@ki.se

Keywords: Congenital heart block, neonatal lupus erythematosus, autoantibodies, SSA, SSB

Word count: 2925 


\section{ABSTRACT}

Objective. Congenital heart block may develop in the fetus of Ro/SSA and La/SSB positive mothers. Reported recurrence rates of only $10-20 \%$ despite persisting maternal antibodies indicate that additional factors are critical for establishment of the block. We therefore investigated the influence of other maternal and fetal factors on heart block development in a Swedish population-based cohort.

Methods. The influence of fetal gender, maternal age, parity and time of birth on heart block development was analyzed in 145 families including Ro/La-positive $(n=190)$ and Ro/Lanegative $(n=165)$ pregnancies.

Results. We observed a recurrence rate of $12.1 \%$ in Ro/La-positive women, and no recurrence in Ro/La-negative women. Fetal gender and parity did not influence the development of heart block in either group. Maternal age in Ro/La-positive pregnancies with a child affected by heart block was however significantly higher than in pregnancies resulting in babies without heart block $(p<0.01)$. Further, seasonal timing of pregnancy influenced the outcome. Gestational susceptibility-weeks 18-24 occurring during January-March correlated with a higher proportion of heart block pregnancies and lower vitamin D levels, and a corresponding higher proportion of children with heart block born in the summer $(p<0.02)$. Maternal age or seasonal timing of pregnancy did not affect the outcome in Ro/La-negative pregnancies.

Conclusion. This study identifies maternal age and seasonal timing of pregnancy as novel risk factors for congenital heart block development in Ro/La positive pregnancy. These observations indicate that the risk can be modified, and will be important for counseling when a pregnancy is considered. 
Congenital complete heart block without cardiac malformation is a rare disease, affecting 1 in 15,000 to 20,000 births in the general population. An association with the presence of maternal autoantibodies to Ro/SSA and/or La/SSB is however well established [1, 2], and the risk of complete congenital heart block is $1-2 \%$ in Ro/SSA-positive pregnancies [3-6]. Furthermore, the reported risk of giving birth to a second child with complete heart block for anti-Ro/SSA positive mothers ranges from 12 to 20\% [7-9], despite the persistence of the maternal autoantibodies [10]. This indicates that additional factors are critical for establishing the heart block. Fetal genetic susceptibility has been suggested as a potential risk factor $[11,12]$, and polymorphisms in the gene encoding TGF $\beta$ have been implicated in the development of heart block $[13,14]$. Variations in the intrauterine environment between pregnancies have also been suggested to contribute to the penetrance of the disease. Maternal disease severity has been investigated as such a potential risk factor, but was not found to contribute to the development of congenital heart block [15]. Given the rarity of congenital heart block occurrence in the general population, it is difficult to investigate potential risk factors associated with the disease. In particular, very little information is available on the influence of maternal age and parity on pregnancy outcome in anti-Ro/La positive mothers. In an effort to address these questions in a reasonable cohort we identified heart block patients in the Swedish population via national health care and hospital registries and investigated maternal and fetal factors associated with congenital heart block in one hundred and forty-five families. Variables analyzed included maternal serological status, age, parity as well as fetal gender and season of birth. 


\section{PATIENTS AND METHODS}

\section{Patients and families}

Identification and enrollment of patients were described in detail previously [16]. Briefly, patients with heart block diagnosed before 15 years of age were identified using the Swedish National Patient Register (www.socialstyrelsen.se) and the Swedish Pacemaker and ICD Register (www.pacemakerregistret.se), as well as by local clinical registers and through a network of pediatric and adult cardiologists and rheumatologists at the six university hospitals in Sweden. Patients with cardiac structural abnormalities as well as patients with post-operative or infection-induced block were excluded from the study. Patients and their families were contacted and offered to take part in the study and donate blood samples. Families for whom a) a blood sample from the mother, b) information on date of birth for the mother and all siblings were available were included in the present study. In total, 145 families including 149 individuals with heart block born between 1949 and 2009, 145 mothers and 208 siblings without heart block were included in the study. The study was approved by the Regional Ethical Committee, Stockholm, Karolinska Institutet, and all participants, or the parents if the individual was $<18$ years, gave informed written consent.

\section{Serologic analyses}

Autoantibodies were detected by Inno-Lia ${ }^{\mathrm{TM}}$ ANA (Innogenetics, Cambereley, United Kingdom) according to the manufacturer's instructions and antibodies to Ro52 further confirmed by ELISA [17].

\section{Vitamin D levels}


For evaluation of vitamin D levels, data were obtained from female Swedish individuals $(n=1068)$, selected as controls from the national population register as described [18]. Vitamin D levels were measured as serum $25(\mathrm{OH})$ vitamin D by a chemiluminescent assay which recognizes $100 \%$ of both $25(\mathrm{OH}) \mathrm{D}_{2}$ and $\left.25(\mathrm{OH}) \mathrm{D}_{3}\right)$ (Liason ${ }^{\circledR}$, DiaSorin, Saluggia, Italy).

\section{Statistical analyses}

Statistical analyses for fetal gender and time of birth were performed using a Chi-square test. Difference in maternal age between antibody-positive or -negative pregnancies leading to the birth of a child with or without heart block was evaluated by Kruskal-Wallis test followed by Dunn's post test. Difference in maternal age at the birth of the first child was evaluated by MannWhitney U-test. The odds ratios (OR) of developing heart block associated with each maternal age category, with and without adjustment for parity, together with $95 \%$ confidence intervals, were calculated by logistic regression. The correlation between vitamin $\mathrm{D}$ levels for each month of the year and the ratio between the numbers of AVB or healthy pregnancies for which the $21^{\text {st }}$ week fell in that particular month was assessed by the non-parametric Spearman correlation test. A $p$ value $<0.05$ was considered significant. Statistical analyses were performed using GraphPad Prism version 5.01 for Windows, GraphPad Software, San Diego, California, USA (www.graphpad.com), as well as Statistica 8.0, Statsoft, Tulsa, OK and SAS software for Windows version V.9.1. 


\section{RESULTS}

\section{Distinction of families with or without maternal autoantibodies}

One hundred and forty-five families with at least one child diagnosed with heart block before the age of fifteen were included in the study. In 80 families $(55 \%)$ the mother carried autoantibodies to Ro and/or La and in 65 families (45\%) the mother had no detectable anti-Ro/La autoantibodies [16]. The presence versus absence of maternal antibodies has been suggested to define two distinct groups of heart block patients regarding pathogenic mechanisms [19], and we therefore investigated the maternal and fetal factors potentially associated with heart block separately in Ro/La-positive $(n=190)$ and Ro/La-negative $(n=165)$ pregnancies.

\section{Recurrence of heart block in subsequent pregnancies}

Of the 80 families enrolled in the study where the mother tested positive for anti-Ro/La antibodies, 33 (41\%) had additional children after the birth of a child with heart block. Of the 33 pregnancies immediately following the birth of the first affected child, 4 resulted in the birth of another child with heart block, generating a recurrence rate of 12.1\% (Figure 1). The overall familial recurrence rate was $8.9 \%$, with 4 affected pregnancies out of a total of 45 including all pregnancies occurring after the birth of a child with heart block.

Of the 65 families where the mother was negative for the presence of anti-Ro/La antibodies, $36(55 \%)$ included one or more siblings born after a child with heart block. However, none of the pregnancies following the birth of the first heart block case was affected, generating a recurrence rate of $0 \%$ in this group (Figure 1). 


\section{Fetal gender of heart block patients versus healthy siblings}

The influence of fetal gender on the development of heart block was next investigated. Female sex was observed in $52 \%$ of the affected children and $56 \%$ of the healthy children born to anti-Ro/La positive mothers, indicating that fetal sex did not predict occurrence of heart block $(p=0.661)$. Similarly, no significant skewing of the gender distribution was observed in the group of children born to anti-Ro/La negative mothers, with $57 \%$ of affected and $53 \%$ of healthy children being female $(p=0.580)$, Table 1 .

\section{Maternal age, but not parity, influences pregnancy outcome in Ro/La-positive mothers}

While the risk of pregnancy complications in general increases with the age of the mother, little is known about the influence of maternal age or parity on pregnancy outcome and heart block development in Ro/La positive women. We therefore investigated the influence of these maternal factors on the development of heart block.

We first analyzed if there was any difference in the age of the mothers in the autoantibody-positive compared to the autoantibody-negative group. The age of the mother at the birth of a child with heart block was significantly higher in anti-Ro/La positive women than in antibody-negative women, 29.5 (28.4-30.6) years; mean (95\% confidence interval), versus 26.9 (25.8-27.9) years respectively, $p<0.01$ (Figure 2A). Since women with anti-Ro/La antibodies represent a group where the prevalence of autoimmune diseases such as Sjögren's syndrome and systemic lupus erythematosus will be much higher than in the group of anti-Ro/La negative women, it is possible that the observed difference in maternal age results from a less favorable health status of antibody-positive women and a delayed maternity in general. However, we did not observe any significant difference in the maternal age at the birth of the first child between antibody-positive and -negative women ( $\mathrm{p}=0.069$, Mann-Whitney U test), Figure 2B. 
We next compared the age of the mothers in Ro/La antibody-positive pregnancies when giving birth to a child with, or without, congenital heart block. Anti-Ro/La positive mothers were significantly older at the time of a pregnancy resulting in a child affected with heart block than at the time of birth of a non-affected baby; 29.5 (28.4-30.6) years, mean ( $95 \%$ confidence interval), versus 27.5 (26.5-28.5) years respectively, $p<0.05$ (Figure 2A). An obvious correlate of increasing age is increasing parity, and to clarify the effect of parity on heart block development we analyzed the risk of having a child with heart block in relation to parity and maternal age. Stratifying the different pregnancies by parity, we observed that anti-Ro/La positive mothers giving birth to a child with heart block were consistently older than antibody-positive women giving birth to a healthy child, independently of whether it was the first, second, third or higher number of pregnancy (Figure 2C and 2D). This trend was not observed in the group of antibodynegative mothers (Figure 2D). Analysis of the influence of maternal age and parity on the risk of having a child with heart block in antibody-positive women revealed that only maternal age had a significant effect $(p=0.01)$ (logistic regression model analysis), while parity did not significantly affect pregnancy outcome $(p=0.35)$.

To estimate the increase in risk due to increasing maternal age, we calculated the odds ratio (OR) for giving birth to a child with heart block in anti-Ro/La antibody-positive women in relation to age (Figure 2E and Table 2). Increasing OR with increasing maternal age was observed, with OR $2.3(1.0-5.5,95 \%$ confidence interval, $\mathrm{p}=0.05)$ and OR $4.2(1.4-11.9, p=$ 0.01 ) for women 30-34 and $\geq 35$ years old, respectively, compared to women $<25$ years of age. In addition, we did not find any evidence of an interaction between maternal age and parity regarding increased odds ratio for the risk of heart block $(p=0.21)$. No significant influence of maternal age or parity was observed in the group of anti-Ro/La antibody negative women (Table 2). 


\section{Season-of-birth influence development of congenital heart block}

Evidence that the time of birth as defined by season or month may influence the development of autoimmune diseases later in life has lately emerged [20]. Heart block in antiRo/La-positive pregnancies usually develops between 18 to 24 weeks of pregnancy, indicating that the pathogenic mechanisms leading to conduction defects in the fetal heart are initiated before and/or within this period of pregnancy. The 18-24 weeks of pregnancy taking place during the late Swedish winter months (January-March) corresponds to a birth occurring during the Swedish summer (June-August), and we hypothesized that events linked to the winter season may affect the outcome of pregnancy and the ratio of healthy and affected births during the summer as compared to the rest of the year.

Analysis of the number of births during the summer (June-August) and the rest of the year revealed a significant difference between affected children and healthy siblings in the Ro/La-positive pregnancy group, with births of children affected by heart block representing $58.5 \%$ of all births in the summer and only $39.0 \%$ of all births during the rest of the year $(p=$ 0.015, Figure 3A). This skewed distribution towards an increase in the proportion of affected births in the summer was not observed in the Ro/La-negative pregnancy group $(p=0.583$, Figure 3B).

The significant increase in the proportion of children with heart block born in the summer as compared to the rest of the year supported our hypothesis that events linked to the winter season and occurring during the susceptibility weeks 18-24 may affect the outcome of the pregnancy. Such a possible factor is the marked decrease in light exposure during winter in Nordic countries like Sweden that may affect vitamin D levels. We therefore analyzed the variation of vitamin D levels over the year based on samples from 1068 Swedish women that had 
been taken at different times of the year. Vitamin D levels were higher during the summer, with the highest mean value observed in August, than during the winter months, with the lowest mean level recorded in March (Figure 3C). We found that average vitamin D levels for each month were significantly correlated to the ratio of heart block to healthy pregnancies for which the median susceptibility week (defined as week 21 of pregnancy) fell in that particular month ( $p=$ 0.009), with low vitamin D levels corresponding to a significantly higher proportion of heart block pregnancies (Figure 3D). 


\section{DISCUSSION}

Isolated complete congenital heart block is well known to associate with the presence of maternal anti-Ro/SSA and La/SSB antibodies. A recurrence rate of $12-20 \%$ however indicates that additional factors are involved in the pathogenic process. The objective of the present study was to investigate the influence of variables such as maternal age, parity, time of birth and fetal gender in the development of heart block. More specifically, we assessed the potential association of these factors with congenital heart block not only by comparing heart block cases to healthy siblings in a cohort of families with autoantibody-positive mothers, but also by analyzing the same variables in a group of heart block patients and healthy siblings born to autoantibody-negative mothers.

In the anti-Ro/La positive group of mothers we observed a recurrence rate of $12.1 \%$ in 33 pregnancies immediately following the birth of a child with heart block, and an overall recurrence rate in all siblings born after an affected child of $8.9 \%$. While low recurrence rates have already been reported [21], recurrence rates of $17 \%$ in pregnancies immediately following the birth of a child with congenital heart block have been observed in large cohorts in two independent studies [8, 22]. However, recurrent cases in both of these studies may be overrepresented as the cohorts were formed either by specifically recruiting families with cases of neonatal lupus [22] or based on registers from tertiary referral centers [8]. By contrast, in this study we analyzed a population-based cohort gathered from national health registers in an effort to minimize the bias towards recruitment of families with multiple heart block cases.

Fetal gender was not associated with development of heart block in Ro/La-positive or negative pregnancies, in accordance with previous reports $[9,22]$. A trend towards higher maternal age for heart block mothers compared to mothers who give birth to healthy babies was 
previously reported by us [23]. In the present study we found that the risk of fetal heart block in Ro/La-positive pregnancies increased significantly with increasing maternal age but was not influenced by parity. This finding was specific for autoantibody-associated heart block, as neither maternal age nor parity influenced the outcome of Ro/La-negative pregnancies.

Complications during pregnancy have been associated with increasing maternal age, affecting both the mother and the child, reviewed in [24]. Disorders such as placental growth retardation and placental abruption are known to increase in frequency with age, mainly due to insufficient utero-placental perfusion. However, there is little information about placental function in relation to maternal age. This factor may be relevant in the context of congenital heart block as hypoxia has been suggested to amplify the harmful effects of anti-Ro/La antibodies in the fetal heart [25]. Hypothyroidism, known to be associated with aging, may also be another factor of interest as anti-Ro positive women with hypothyroidism have been shown to be at higher risk of having a child with complete congenital heart block compared to women with autoantibodies alone [26]. It is also possible that the increasing risk for heart block with increasing maternal age reflects the appearance of anti-Ro/La autoantibodies in women over time. This hypothesis could however not be addressed in the present study, as blood samples from mothers were only collected after the birth of a child with heart block.

Seasonal variation in the time of birth has recently been associated with the development of autoimmune diseases later in life, eg multiple sclerosis [20]. We speculated that events such as low wintertime vitamin D levels at the critical period of potential heart block development in fetuses of women with anti-Ro/La autoantibodies might affect the outcome of the pregnancy. This specific time window (18-24 weeks of gestation) during the winter corresponds to birth in the summer. In the present study we found that the distribution of heart block births over the year was skewed towards an increased proportion of affected children born in the summer. 
Additionally, we found that vitamin D levels, which varied in a Swedish female population over the year in relation to the seasons, were inversely correlated to the number of heart block pregnancies in autoantibody-positive women. Although the vitamin D levels used in this study originated from a population of healthy Swedish women and not from the specific mothers of our cohort, it is reasonable to believe that these women would follow the same general trend. Despite the well-documented immunomodulatory role of vitamin D (reviewed in [27]), its potential relation to the development of heart block in utero remains to be elucidated. While the association between the winter season, decreased sun exposure and vitamin D levels comes readily to mind, other events linked to the winter season such as viral infections might also influence the development of heart block.

In summary, we report a recurrence rate of $12.1 \%$ in anti-Ro/La positive pregnancies and demonstrate a significant association of increased maternal age and birth in the summer with development of heart block in antibody-positive pregnancies. These associations were not observed in antibody-negative pregnancies. Our data support the idea of different pathogenic mechanisms of heart block in anti-Ro/La positive and negative pregnancies, and are also of great interest to improve the counseling of antibody-positive women contemplating pregnancy. 
Table 1. Association between fetal gender and pregnancy outcome.

\begin{tabular}{|c|c|c|c|c|}
\hline & $\begin{array}{c}\text { Total } \\
\mathrm{n}\end{array}$ & $\begin{array}{l}\text { Female } \\
\text { n (\%) }\end{array}$ & $\begin{array}{l}\text { Male } \\
\text { n (\%) }\end{array}$ & $p^{*}$ \\
\hline \multicolumn{5}{|c|}{$\mathrm{Ro} / \mathrm{La}+$ pregnancies } \\
\hline \multirow[t]{2}{*}{ AVB } & 84 & $44(52)$ & $40(48)$ & \multirow{3}{*}{0.661} \\
\hline & & & & \\
\hline Healthy & 108 & $60(56)$ & $48(44)$ & \\
\hline \multicolumn{5}{|c|}{ Ro/La- pregnancies } \\
\hline \multirow[t]{2}{*}{ AVB } & 65 & $37(57)$ & $28(43)$ & \multirow{3}{*}{0.580} \\
\hline & & & & \\
\hline Healthy & 99 & $52(53)$ & $47(47)$ & \\
\hline
\end{tabular}

* $p$ values were determined by the Chi-square test. 
Table 2. Odds ratio of fetal heart block in relation to maternal age.

\begin{tabular}{|c|c|c|c|c|c|}
\hline & Age & OR $(95 \% \mathrm{CI})$ & $p$ & OR $(95 \% \mathrm{CI})$ & $p$ \\
\hline & & & & \multicolumn{2}{|c|}{ Adjusted for parity } \\
\hline \multirow[t]{4}{*}{$\mathrm{Ro} / \mathrm{La}+$ pregnancies } & $\leq 24$ & 1.0 (Reference) & Reference & 1.0 (Reference) & Reference \\
\hline & $25-29$ & $1.7(0.8-3.7)$ & 0.18 & $1.6(0.7-3.6)$ & 0.22 \\
\hline & $30-34$ & $2.0(0.9-4.5)$ & 0.09 & $2.3(1.0-5.5)$ & 0.05 \\
\hline & $\geq 35$ & $3.2(1.2-8.4)$ & 0.02 & $4.2(1.4-11.9)$ & 0.01 \\
\hline \multirow[t]{4}{*}{ Ro/La- pregnancies } & $\leq 24$ & 1.0 (Reference) & Reference & 1.0 (Reference) & Reference \\
\hline & $25-29$ & $0.8(0.4-1.6)$ & 0.46 & $0.9(0.4-1.9)$ & 0.77 \\
\hline & $30-34$ & $1.1(0.4-2.6)$ & 0.90 & $1.7(0.6-4.9)$ & 0.30 \\
\hline & $\geq 35$ & $0.4(0.1-1.6)$ & 0.20 & $0.9(0.2-4.4)$ & 0.90 \\
\hline
\end{tabular}




\section{ACKNOWLEDGEMENTS}

We thank Drs. Paul Blomqvist, Department of Medicine, Karolinska Institutet and Solveig Wållberg-Jonsson, Department of Rheumatology, Umeå University Hospital for valuable discussions and case contribution, respectively.

\section{COMPETING INTERESTS}

None.

\section{FUNDING}

Financial support for this study was obtained from KIRCNET (Karolinska Institutet Circulation and Respiratory Research Network), the Magn. Bergvalls Foundation, the Jerring Foundation, Stiftelsen Samariten, the Karolinska Institute, The Royal Swedish Academy of Sciences, the Swedish Research Council, the Göran Gustafsson Foundation, the Torsten and Ragnar Söderberg Foundation, the King Gustaf the V:th 80-year foundation, the Swedish Foundation for Strategic Research, the Heart-Lung Foundation and the Swedish Rheumatism Association. 


\section{REFERENCES}

1 Salomonsson S, Strandberg L. Autoantibodies associated with congenital heart block. Scand J Immunol 2010;72:185-188.

2 Brucato A, Previtali E, Ramoni V, et al. Arrhythmias presenting in neonatal lupus. Scand J Immunol 2010;72:198-204.

3 Cimaz R, Spence DL, Hornberger L, et al. Incidence and spectrum of neonatal lupus erythematosus: a prospective study of infants born to mothers with anti-Ro autoantibodies. J Pediatr 2003;142:678-683.

4 Costedoat-Chalumeau N, Amoura Z, Lupoglazoff JM, et al. Outcome of pregnancies in patients with anti-SSA/Ro antibodies: a study of 165 pregnancies, with special focus on electrocardiographic variations in the children and comparison with a control group. Arthritis Rheum 2004;50:3187-3194.

5 Brucato A, Frassi M, Franceschini F, et al. Risk of congenital complete heart block in newborns of mothers with anti-Ro/SSA antibodies detected by counterimmunoelectrophoresis: a prospective study of 100 women. Arthritis Rheum 2001;44:1832-1835.

6 Hornberger LK, Al Rajaa N. Spectrum of cardiac involvement in neonatal lupus. Scand J Immunol 2010;72:189-197.

7 Buyon JP, Hiebert R, Copel J, et al. Autoimmune-associated congenital heart block: demographics, mortality, morbidity and recurrence rates obtained from a national neonatal lupus registry. J Am Coll Cardiol 1998;31:1658-1666.

8 Julkunen $\mathrm{H}$, Eronen M. The rate of recurrence of isolated congenital heart block: a population-based study. Arthritis Rheum 2001;44:487-488. 
9 Solomon DG, Rupel A, Buyon JP. Birth order, gender and recurrence rate in autoantibody-associated congenital heart block: implications for pathogenesis and family counseling. Lupus 2003;12:646-647.

10 Strandberg L, Salomonsson S, Bremme K, et al. Ro52, Ro60 and La IgG autoantibody levels and Ro52 IgG subclass profiles longitudinally throughout pregnancy in congenital heart block risk pregnancies. Lupus 2006;15:346-353.

11 Strandberg LS, Ambrosi A, Jagodic M, et al. Maternal MHC regulates generation of pathogenic antibodies and fetal MHC-encoded genes determine susceptibility in congenital heart block. J Immunol 2010;185:3574-3582.

12 Wahren-Herlenius M, Sonesson SE. Specificity and effector mechanisms of autoantibodies in congenital heart block. Curr Opin Immunol 2006;18:690-696.

13 Clancy RM, Backer CB, Yin X, et al. Cytokine polymorphisms and histologic expression in autopsy studies: contribution of TNF-alpha and TGF-beta 1 to the pathogenesis of autoimmune-associated congenital heart block. J Immunol 2003;171:3253-3261.

14 Cimaz R, Borghi MO, Gerosa M, et al. Transforming growth factor beta1 in the pathogenesis of autoimmune congenital complete heart block: lesson from twins and triplets discordant for the disease. Arthritis Rheum 2006;54:356-359.

15 Eronen M, Miettinen A, Walle TK, et al. Relationship of maternal autoimmune response to clinical manifestations in children with congenital complete heart block. Acta Paediatr 2004;93:803-809.

16 Zeffer E, Salomonsson S, Dzikaite V, et al. A population-based investigation of the autoantibody profile in mothers of children with atrioventricular block [abstract]. Scand J Immunol 2010;72:274-275. 
17 Salomonsson S, Dorner T, Theander E, et al. A serologic marker for fetal risk of congenital heart block. Arthritis Rheum 2002;46:1233-1241.

18 Hedstrom AK, Baarnhielm M, Olsson T, et al. Tobacco smoking, but not Swedish snuff use, increases the risk of multiple sclerosis. Neurology 2009;73:696-701.

19 Villain E, Coastedoat-Chalumeau N, Marijon E, et al. Presentation and prognosis of complete atrioventricular block in childhood, according to maternal antibody status. J Am Coll Cardiol 2006;48:1682-1687.

20 Willer CJ, Dyment DA, Sadovnick AD, et al. Timing of birth and risk of multiple sclerosis: population based study. BMJ 2005;330:120.

21 Julkunen $\mathrm{H}$, Kaaja R, Wallgren E, et al. Isolated congenital heart block: fetal and infant outcome and familial incidence of heart block. Obstet Gynecol 1993;82:11-16.

22 Llanos C, Izmirly PM, Katholi M, et al. Recurrence rates of cardiac manifestations associated with neonatal lupus and maternal/fetal risk factors. Arthritis Rheum 2009;60:3091-3097.

23 Skog A, Wahren-Herlenius M, Sundstrom B, et al. Outcome and growth of infants fetally exposed to heart block-associated maternal anti-Ro52/SSA autoantibodies. Pediatrics 2008;121:e803-809.

24 Montan S. Increased risk in the elderly parturient. Curr Opin Obstet Gynecol 2007;19:110-112.

25 Clancy RM, Zheng P, O'Mahony M, et al. Role of hypoxia and cAMP in the transdifferentiation of human fetal cardiac fibroblasts: implications for progression to scarring in autoimmune-associated congenital heart block. Arthritis Rheum 2007;56:4120-4131. 
26 Spence D, Hornberger L, Hamilton R, et al. Increased risk of complete congenital heart block in infants born to women with hypothyroidism and anti-Ro and/or anti-La antibodies. J Rheumatol 2006;33:167-170.

27 Mora JR, Iwata M, von Andrian UH. Vitamin effects on the immune system: vitamins A and D take centre stage. Nat Rev Immunol 2008;8:685-698. 


\section{FIGURE LEGENDS}

Figure 1. Family size and birth order of heart block cases. Children with heart block are represented by solid black circles, children without heart block are represented by open circles. A solid grey circle represents twins without heart block.

Figure 2. Development of heart block in Ro/La-positive pregnancies is associated with increased maternal age but independent of parity. A. Maternal age at the birth of a child with or without AVB in anti-Ro/La positive or negative pregnancies. B. Maternal age at the birth of the first child for antibody-positive and -negative women. $(\mathrm{A}, \mathrm{B})$ Data shown as box plots $\left(25^{\text {th }}\right.$ to $75^{\text {th }}$ percentiles) with line at median and whiskers at minimum and maximum. C. Age of antibody-positive women at the birth of their children, grouped by parity (first to sixth child) and outcome (AVB or no AVB). Line at mean. D. Maternal age at the birth of a child depending on antibody status (anti-Ro/La positive or negative) and pregnancy outcome (AVB or no AVB) presented as the mean with its $95 \%$ confidence interval. E. Graphic representation of odds ratio (OR) with $95 \%$ confidence interval for giving birth to a child with heart block in autoantibodypositive women dependent on maternal age and adjusted for parity. The reference group corresponds to maternal age $\leq 24$ years ( $n=16$ AVB, 35 no AVB); 25-29 years $n=28$ AVB, 36 no AVB ; 30-34 years $n=24$ AVB, 26 no AVB ; $\geq 35$ years $n=16$ AVB, 11 no AVB. ${ }^{*} p<0.05, * * p$ $<0.01$.

Figure 3. Development of heart block shows a season-of-birth pattern and correlates to vitamin D levels during the susceptibility weeks 18-24 of pregnancy. A,B. Proportions of affected (grey fields) and healthy (white fields) births during summer and the rest of the year for 
anti-Ro/La positive (A) and anti-Ro/La-negative (B) mothers represented as percentage of the total number of births during the respective periods. The number (n) of births in each group is indicated. C. Vitamin D levels (mean, SEM) in a group of healthy Swedish women $(n=1068)$, pooled by month according to blood sampling time. D. Correlation between the average vitamin $\mathrm{D}$ levels for each month (mean $\pm \mathrm{SEM}$ ) and the ratio of the number of AVB to the number of healthy pregnancies for which week 21 of pregnancy falls in that particular month. $p$-value calculated by the Spearman correlation test. 
$\mathrm{Ro} / \mathrm{La}+$ mothers

No. of families

Family size, birth- $\quad$ No. of families

order case pattern

exhibiting case
Ro/La - mothers

No. of families

No. of families pattern of each size exhibiting case pattern 1-child

$\bullet$

2-child

- 0

O

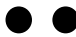

3-child

- 0 ०

$0 \bullet 0$

0

O

00

O

4-child

- 000

000

0000

000

6-child

- 00000

000000

000000
11

11

36

9

24

3

27

6

8

1

1

10

1

4

0

1

2

1

2
19

9

30

17

9

5

0

5

0

2

3

3

1

1
13

0

6

0


A

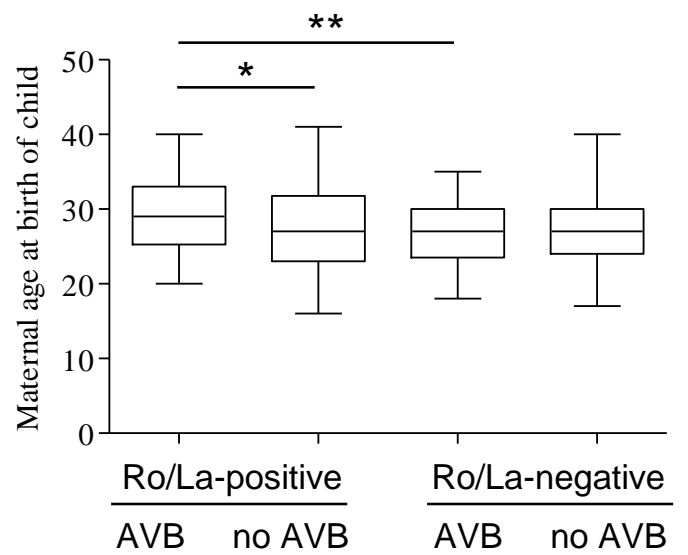

B

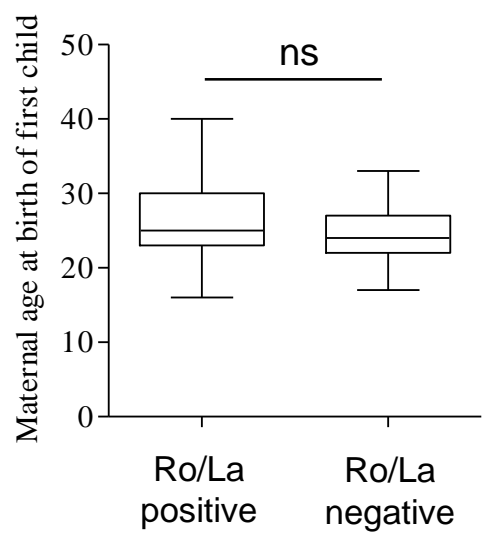

C

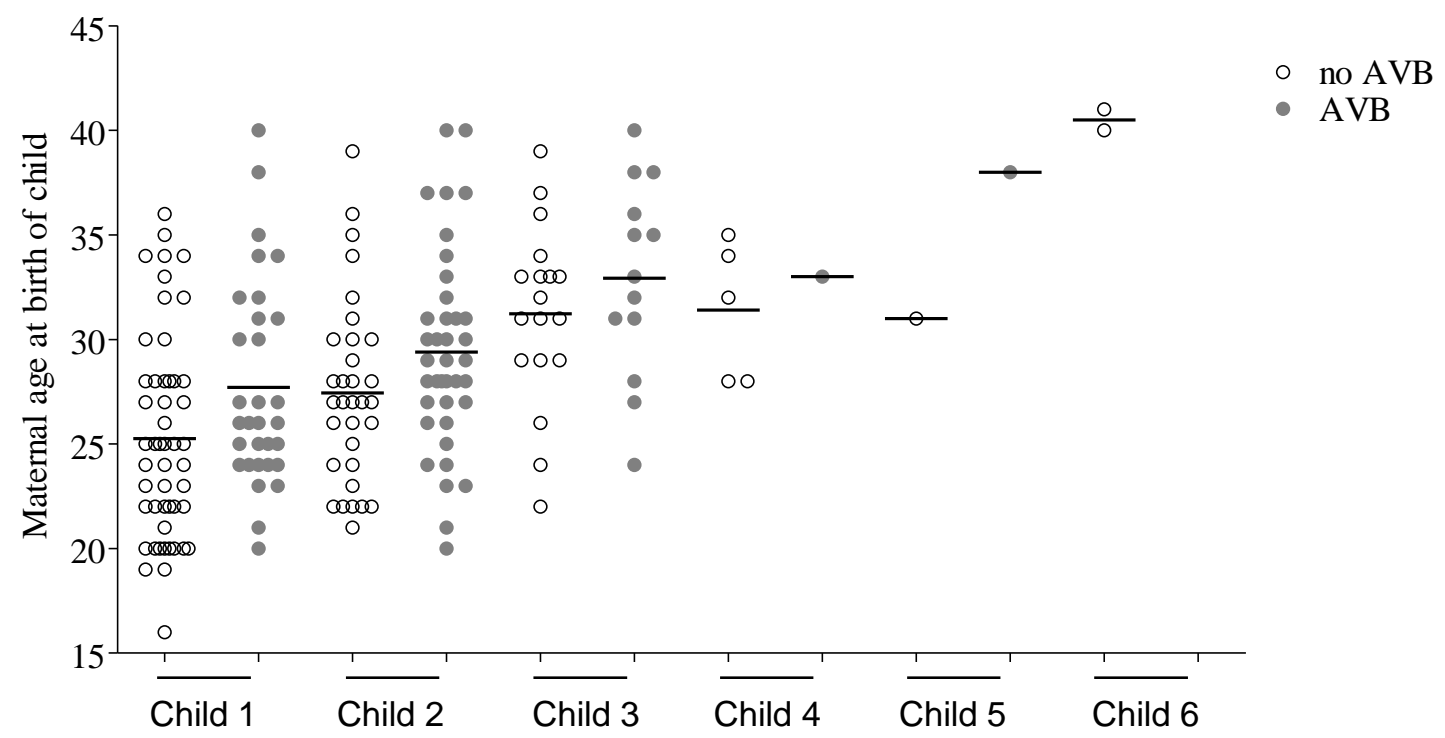

D

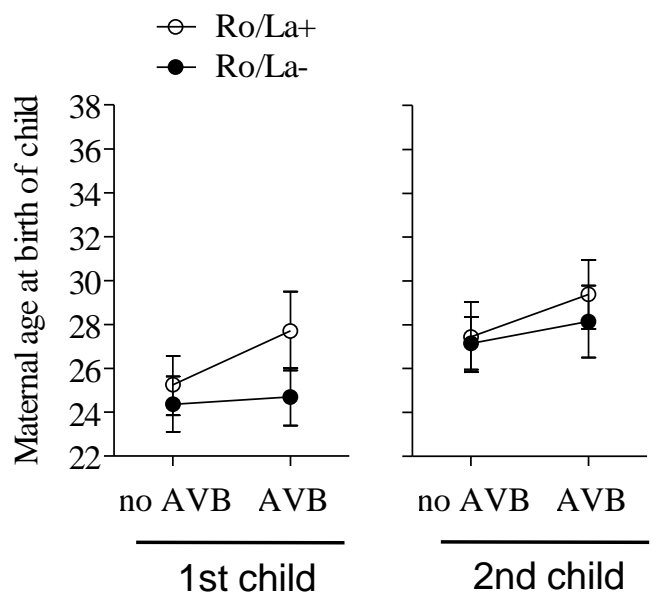

E $\left.\quad{ }^{12}\right]$
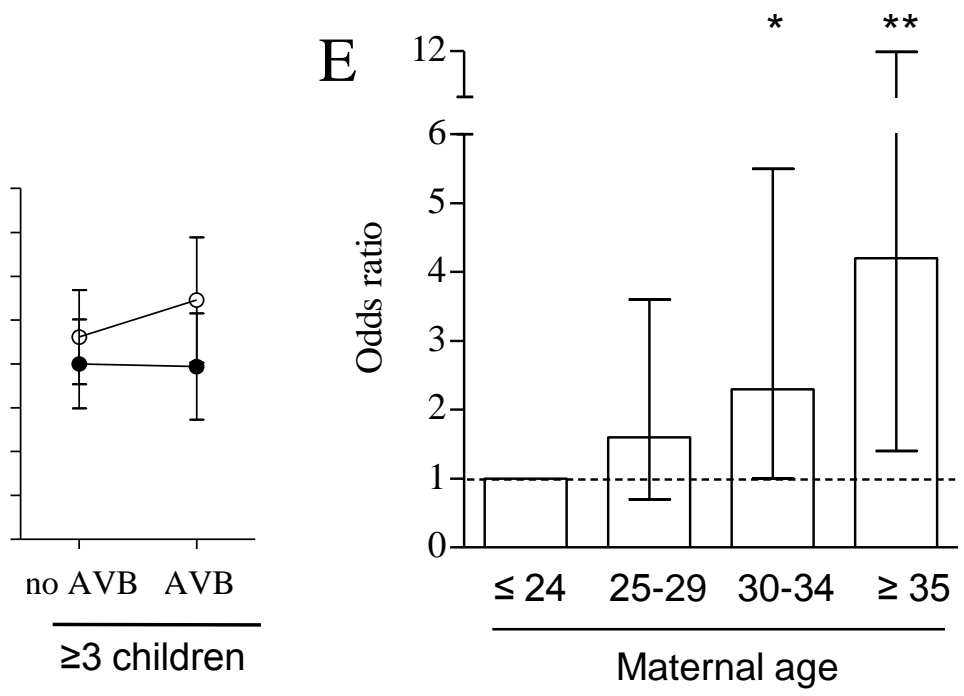

Figure 2, Ambrosi et al 
A

Ro/La-positive pregnancies

$$
p=0.015
$$

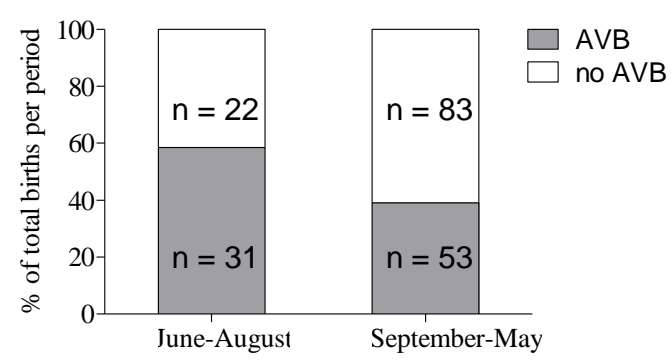

C

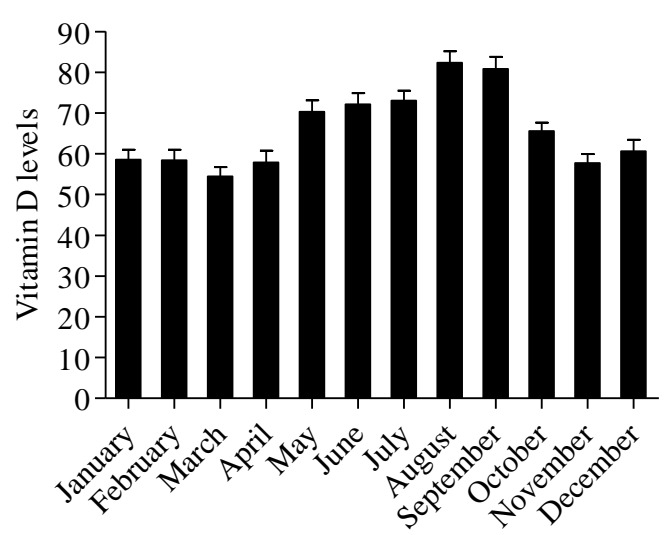

B Ro/La-negative pregnancies

$$
p=0.583
$$

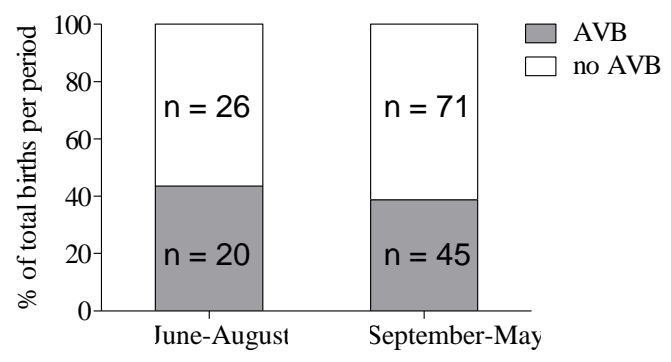

D

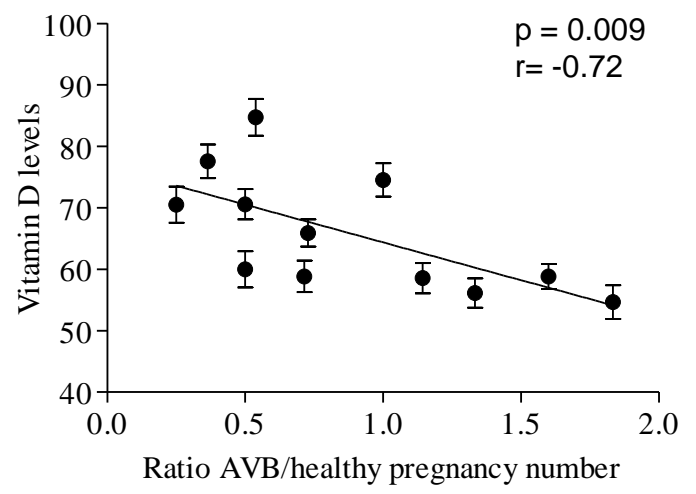

Figure 3, Ambrosi et al 